\title{
Design and Implementation of Water Quality Monitoring System for Agricultural Irrigation Water
}

\author{
Jie Huang \\ College of Mechanical and Electronic Engineering, Hezhou University, Hezhou, China, 542899 \\ huangjie0773@163.com
}

Keywords: Water quality monitoring; Zigbee; GPRS; Irrigation water

\begin{abstract}
In order to monitor the water quality of agricultural irrigation water conveniently and accurately, based on the actual demand of the agricultural irrigation water quality monitoring system, a water quality monitoring system for agricultural irrigation water was studied and designed based on the Zigbee technology and GPRS technology. The structure and composition of the system, the software design of the system, the hardware design of the system, the data fusion algorithm of the monitoring node and the data fusion algorithm of the router node are described in this paper. The system has been tested. The results show that the function and performance index of the system meet the design requirements. It has the advantages of good stability and high cost performance. It has good application prospects.
\end{abstract}

\section{Introduction}

The growth and yield of crops are closely related to the water quality of irrigation water [1,2]. The $\mathrm{pH}$ value, temperature and turbidity of irrigation water have influence on the growth of crops. The roots of crops need to be in a range of $\mathrm{pH}$ value to carry out the normal metabolism. The $\mathrm{pH}$ value of irrigation water affects the effective release of nutrient elements in the culture medium. Different crops for irrigation water $\mathrm{pH}$ value of the requirements are not the same. The temperature of irrigation water and the turbidity of irrigation water affect the absorption and transformation of soil minerals in crops. It is very important to monitor the water quality of agricultural irrigation water conveniently and accurately, which is very important to promote the growth of crops and increase the yield of crops. Zigbee technology is a kind of low speed, short distance and low power consumption of wireless communication technology. It supports tree structure, star network structure and network structure. Zigbee technology has been widely used in forest fire monitoring, flood monitoring, environmental monitoring and precision agriculture [3,4]. If the Zigbee technology is used to monitor the water quality of agricultural irrigation water, it can not only monitor the water quality of irrigation water easily and accurately, but also it has the advantages of convenient wiring and good expansibility [5].

\section{System Structure}

The water quality monitoring system of agricultural irrigation water was studied and designed by using Zigbee technology. It consists of monitoring node, router node, coordinator node, local monitoring center and remote monitoring center. The structure of the system is shown in Fig. 1. The monitoring node is designed by Zigbee technology. It can monitor the water quality parameters such as $\mathrm{pH}$ value, temperature and turbidity of irrigation water. It can also use the Zigbee technology for networking, communication and transmission of data. Monitoring node, router node and coordinator node using Zigbee technology to build a Zigbee wireless sensor network. The local monitoring center is connected with the coordinator through the serial port. The remote monitoring center is connected with the coordinator through the GSM network. 


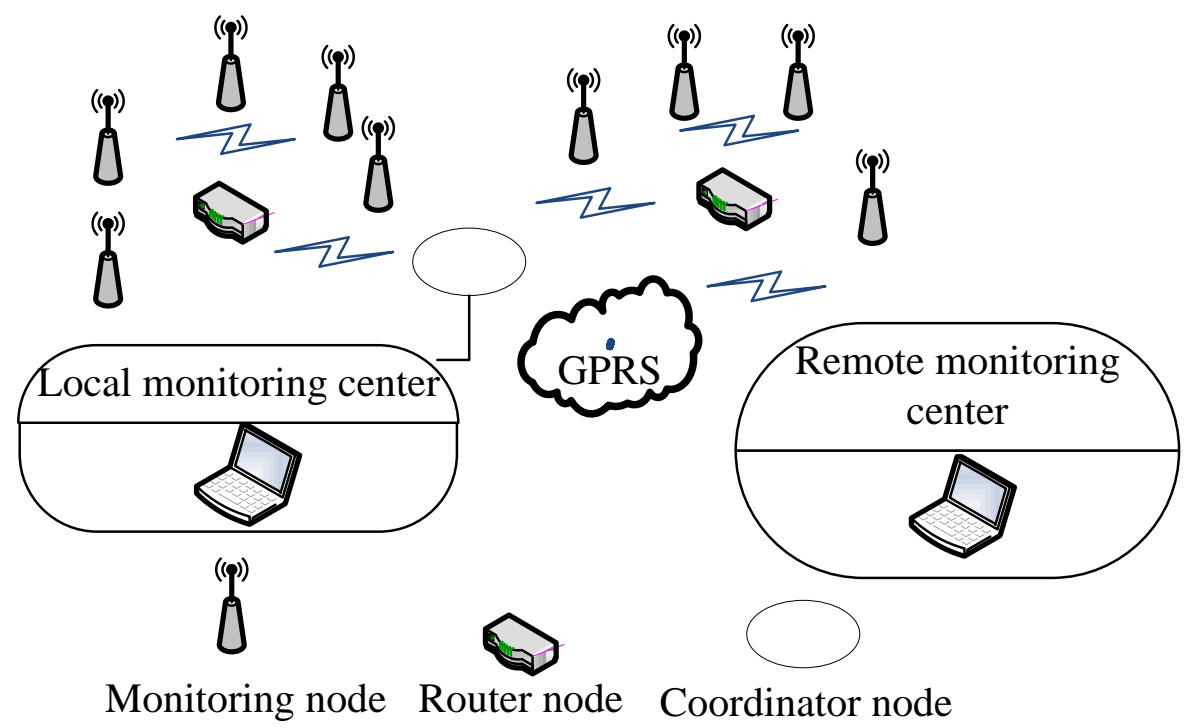

Figure 1. System structure

\section{System Design}

The hardware design of the system includes the hardware design of the monitoring node, the hardware design of the router node and the hardware design of the coordinator node. Limited to the length of the article, it only introduces the hardware design of the $\mathrm{pH}$ value detection circuit.

The $\mathrm{pH}$ value of irrigation water is detected by $\mathrm{pH}$ value detection circuit. The $\mathrm{PH}$ value detection circuit includes measuring electrode and $\mathrm{pH}$ value signal processing circuit. The common $\mathrm{pH}$ value measuring electrode has the glass electrode and the metal electrode. The glass electrode has the characteristics of high resistance, poor anti-interference ability and wide measurement range of $\mathrm{pH}$ value. The metal electrode has the characteristics of small resistance, strong anti-interference ability and narrow measurement range of $\mathrm{pH}$ value [6,7]. In this paper, measuring electrode uses glass electrode. If the $\mathrm{pH}$ value of the irrigation water is alkaline, the output of the glass electrode is negative voltage. If the $\mathrm{pH}$ value of irrigation water is acidic, the output of the glass electrode is positive voltage. $\mathrm{PH}$ value of the signal processing circuit is shown in Fig. 2. Because of the larger resistance glass electrode, the first stage amplifier circuit must have a larger input resistance value. In order to ensure that the output signal is a positive voltage, the input voltage of the same input terminal of the operational amplifier in the output stage is set to be reasonable. The voltage gain of the $\mathrm{pH}$ value signal processing circuit can be adjusted by the potentiometer $\mathrm{R} 2$ and the potentiometer R9. The input voltage of the same input terminal of the operational amplifier in the output stage can be adjusted by the potentiometer R6.

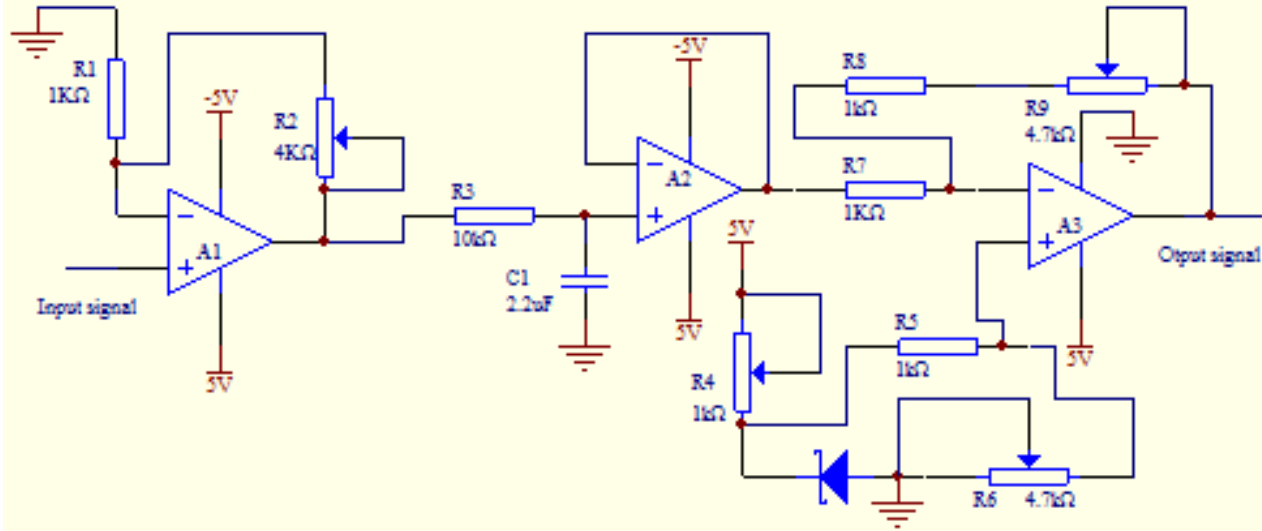

Figure 2. PH signal processing circuit 
Data Fusion Algorithm of Monitoring Node. In Zigbee wireless sensor networks, the nodes are usually powered by batteries. Nodes usually work in unattended environments. Reducing the energy consumption of node has a very important role for the stability of the network and the extension of the working time of the nodes. Node's energy consumption includes the calculation of energy consumption and communication energy consumption. In the energy consumption of nodes, communication energy consumption is the highest proportion. The total energy consumption of the node can be reduced effectively by reducing the communication energy consumption of the node $[8,9]$. In order to effectively reduce the energy consumption of the monitoring node, the data fusion algorithm is used to deal with the data of the monitoring node. And then decide whether to send the data according to the processing results. The data fusion algorithm of the monitoring node is shown below.

1) It assumes that there are $\mathrm{N}$ sensors in the monitoring node. Each sensor has a unique number.

2) It is assume that $D_{24}$ is the four data collected by the sensor, and the number of the sensors is two. $D_{21}, D_{22}$ and $D_{23}$ are stored in an array A, and it has been arranged in order from small to large. The length of the array $\mathrm{A}$ is assumed to be $\mathrm{m}$.

3) If the Eq.1 and the Eq. 2 are simultaneously satisfied, compare the size of $\mathrm{k}$ and $\mathrm{m}$. According to the requirements of the system for monitoring data, $\alpha$ can be flexibly set.

(1) If $\mathrm{k}$ is greater than $\mathrm{m}$, all the data in the array $\mathrm{A}$ is set to $0 . D_{2 k}$ is saved in $\mathrm{A}[0]$. Variable $\mathrm{k}$ is set to 1 .

(2) If $\mathrm{k}$ is less than or equal to $\mathrm{m}, D_{2 k}$ is saved to the array A. The data in the array A is arranged and stored in the order of small to large.

$$
\begin{aligned}
& \left|D_{2 k}-\mathrm{A}[0]\right| \leq \alpha \\
& \left|D_{2 k}-\mathrm{A}[k-2]\right| \leq \alpha
\end{aligned}
$$

4) If the Eq.3 or Eq.4 is satisfied, $\bar{a}$ is calculated by the Eq.5. All the data in the array A is set to 0 . Variable $\mathrm{k}$ is set to 0 . The $\bar{a}$ will be sent to the router node through the Zigbee wireless network.

$$
\begin{aligned}
& \left|D_{2 k}-\mathrm{A}[0]\right|>\alpha \\
& \left|D_{2 k}-\mathrm{A}[k-2]\right|>\alpha \\
& \bar{a}=\frac{1}{k}\left(\sum_{j=0}^{k-2} A[j]+D_{2 k}\right)
\end{aligned}
$$

5) Repeat 2) 4).

Data Fusion Algorithm of Router Node. Monitoring data of the monitoring node is sent to the router node through Zigbee wireless network. Due to the influence of the external environment and the sensor measurement error, there is a certain error in the monitoring data of the same type of sensor in different monitoring nodes $[10,11]$. The error size of each sensor's monitoring data is inconsistent. In order to improve the accuracy of the monitoring data, the data of the router node is processed by the data fusion algorithm. The data fusion algorithm of the router node is shown below.

1) It assumes that a router node has $\mathrm{K}$ monitoring nodes connected with it. Every monitoring node has a unique number. Every monitoring node has $\mathrm{N}$ sensors connected with it. According to the type of sensor, the sensors of in the monitoring node are numbered. In different monitoring nodes, the same type of sensor has the same number.

2) If the number of monitoring node is $M$, and the number of the sensor is $I$, then the sensor is sent to the router node data is $R_{M I}$. 
3) It assumes that $W_{M I}$ is the weight coefficient of a sensor. The number of the sensor is I. The number of monitoring node is $\mathrm{M}$. The weight coefficient of the sensor is calculated according to the Eq. 6.

$$
W_{M I}=\frac{R_{M I}-\frac{1}{K} \sum_{J=1}^{K} R_{J I}}{\frac{1}{K} \sum_{J=1}^{K} R_{J I}}
$$

$$
\sum_{J=1}^{K} W_{J I}=1
$$

4) If the $R_{M I}$ is updated, $\bar{R}_{M I}$ is calculated according to the Eq.8.

$$
\bar{R}_{M I}=\sum_{M=1}^{K} W_{M I} \cdot R_{M I}
$$

5) The $\bar{R}_{M I}$ will be sent to the coordinator node through the Zigbee wireless network.

6) Repeat 3) 5).

Program Design Flow Chart of Monitoring Node. The data of each sensor is collected regularly by the monitoring node. Data is analyzed, calculated and stored by the monitoring node. The program design flow chart of the monitoring node is shown in Fig. 3.

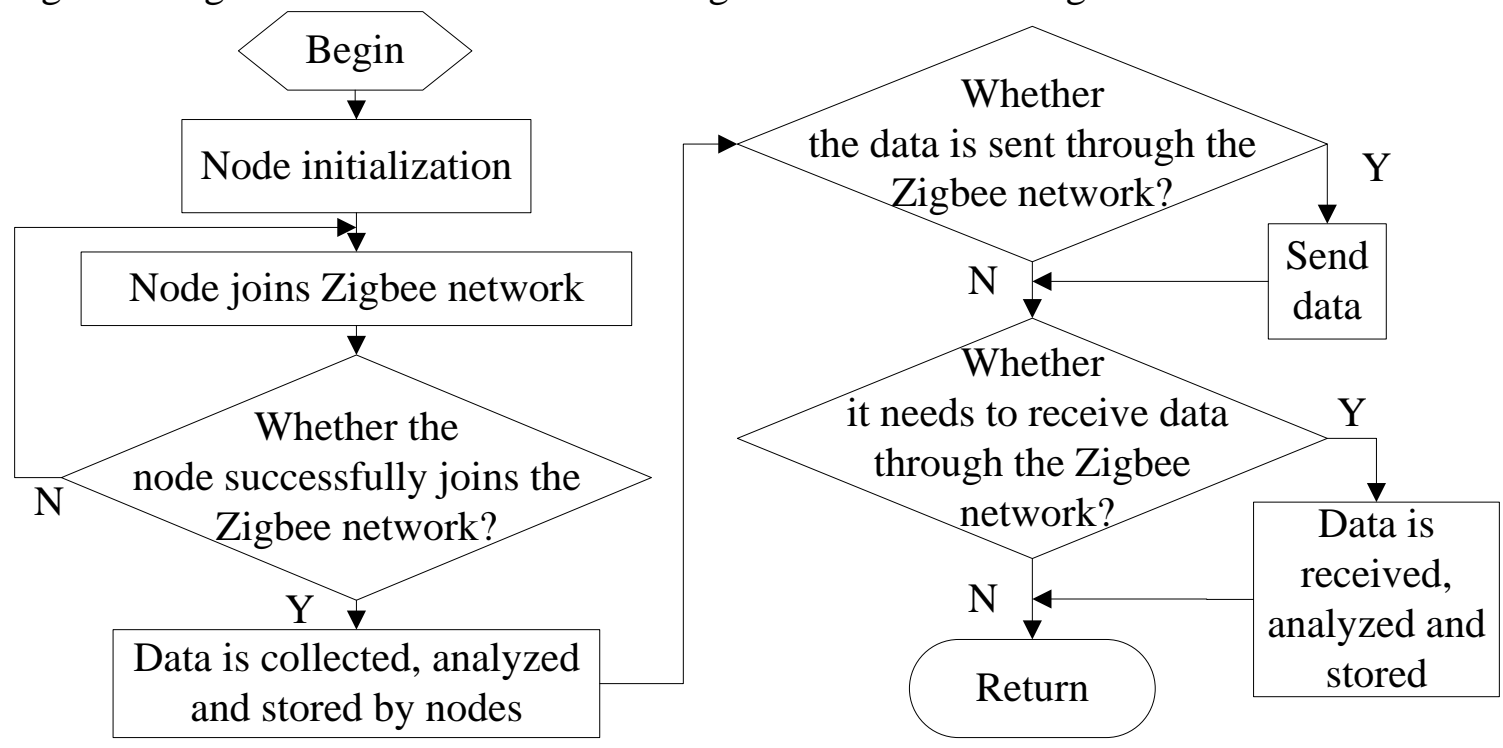

Figure 3. Program design flow chart of monitoring node

\section{System Test}

In order to verify the function and feasibility of the system, it has been installed in an agricultural irrigation area. The function and performance index of the system are tested. The test data are shown in Table 1. The monitoring data in Table 1 is the monitoring data of the system. The measured data in Table 1 is measured by the related instruments. The relative error of $\mathrm{pH}$ value is $2.56 \%$. The absolute error of the $\mathrm{pH}$ value is 0.2 . The relative error of temperature is $0.7 \%$. The absolute error of the temperature value is 0.2 . The relative error of turbidity is $0.42 \%$. The absolute error of the turbidity value is 0.1 . Test results show that the functions and performance of the system indicators meet the design requirements. 
Table 1 System test data

\begin{tabular}{ccccc}
\hline Test item & Monitoring data & Measured data & Relative error & Absolute error \\
\hline PH value & 7.6 & 7.8 & $2.56 \%$ & 0.2 \\
Temperature & $28.5\left[{ }^{\circ} \mathrm{C}\right]$ & $28.3\left[{ }^{\circ} \mathrm{C}\right]$ & $0.7 \%$ & 0.2 \\
Turbidity & $23.7[$ degrees] & $23.8[$ degrees] & $0.42 \%$ & 0.1 \\
\hline
\end{tabular}

\section{Summary}

In order to monitor the water quality of agricultural irrigation water accurately and conveniently, a water quality monitoring system for agricultural irrigation water was studied and designed based on the Zigbee technology and GPRS technology. It consists of monitoring node, router node, coordinator node, local monitoring center and remote monitoring center. In order to effectively reduce the energy consumption of the monitoring node, the data fusion algorithm is used to deal with the data of the monitoring node. It can effectively reduce the energy consumption of the monitoring node. The data fusion algorithm is used to process the data in the router node. It effectively improves the accuracy of monitoring data. After testing, the system has good stability and high cost performance. It has good application prospects.

\section{Acknowledgement}

This work was financially supported by the National Natural Science Foundation of China (Grant No.6154055), the Doctor's scientific research foundation of Hezhou University (No.HZUBS201506).

\section{References}

[1] W.H. Wang, W.G. Yue, Y.F. Wang, W.H. Gu and T.F. Zhao: Electronic Design Engineering, Vol. 24 (2016) No.7, p.135. (In Chinese)

[2] S.J. Pu, Y.S. Wang, H.X. Zhang and X.F. Yang: Journal of Gansu Sciences, Vol. 28 (2016) No.1, p.55. (In Chinese)

[3] H. Pan, J.N. Guan and T.H. Li: Journal of Chinese Agricultural Mechanization, Vol. 35 (2014) No.5, p.246. (In Chinese)

[4] J.Q. Huang, W.X. Wang, S. Jiang, D.Z. Sun, G.C. Ou and K.J. Lu: Transactions of the Chinese Society of Agricultural Engineering, Vol. 29 (2013) No.4, p.183. (In Chinese)

[5] X.Q. Wang, Y.J. Ou and N.L. Huang: Design and Implementation of ZigBee Wireless Sensor Network (Chemical Industry Press, Chinese 2012), p.125. (In Chinese)

[6] Y.H. Li, M.Z. Li, X.L. Deng, H. Sun and X.D. Yang: Transactions of the Chinese Society of Agricultural Engineering, Vol. 29 (2013) No.9, p.170. (In Chinese)

[7] J.F. Li, F.X. Liu, Z.H. Yang and X.D. Yang: Computer Measurement \& Control, Vol. 22 (2014) No.12, p.3887. (In Chinese)

[8] L.M. Sun, J.Z. Li, Y. Chen and H.S. Zhu: Wireless Sensor Network (Tsinghua University Press, Chinese 2005), p.126. (In Chinese)

[9] H.B. Yu, W. Liang and P. Zeng: Intelligent Wireless Sensor Network System (Science Press, Chinese 2013), P.50. (In Chinese)

[10]X.Q. Zhao, S.W. Chen and P.B. Zhang: Computer Measurement \& Control, Vol. 23 (2015) No.11, p.3627. (In Chinese)

[11]Y.G. Wang and Z. Wang: Wireless Sensor Networks (Publishing House of Electronics Industry, Chinese 2012), p.58. (In Chinese) 